\title{
Resection of Giant Tumor of Chest Wall under Cardiopulmonary Bypass after Neoadjuvant Chemoradiotherapy: A Case Report
}

\author{
Lifei Meng, ${ }^{1}$ Yang Wang, ${ }^{1}$ Wang Lei, ${ }^{2}$ Zhigang Liang, ${ }^{1}$ Chenwei Li, ${ }^{1}$ Xinjian $\mathrm{Li}^{1}$ \\ ${ }^{1}$ Department of Thoracic Surgery, Ningbo First Hospital, Ningbo, Zhejiang, China; \\ ${ }^{2}$ Department of Cardiothoracic Surgery, Xinhua Hospital, Shanghai Jiaotong University School of Medicine
}

\section{ABSTRACT}

Osteosarcoma, one of the most common sources of bone malignant tumors, often occurs at the epiphysis, such as in the distal femoral and proximal tibia, but seldom occurs at the costa. Here, we present the case of a 15 -year-old girl with giant osteosarcoma on the chest wall. Osteosarcoma is extremely malignant and has a high death rate. We surgically resected the osteosarcoma using cardiopulmonary bypass and administered neoadjuvant chemoradiotherapy.

\section{INTRODUCTION}

Osteosarcoma is the most common type of primary bone cancer, and the tumor cells directly form osteoid tissues or a bone substance [Zhang 2021]. The cancer, which is most common among 11- to 30-year-old individuals, often occurs at the epiphysis, such as in the distal femoral and proximal tibia, but seldom occurs at the costa [Anoop 2014; Haridas 2020]. Osteosarcoma of the costa accounts for $<3 \%$ of cases and can be completely removed via surgery only [Sabanathan 1997; Ogundiran 2006]. Researchers have reported that rib osteosarcoma, having a poor prognosis [Bacci 2010], comprehensively depends on surgical resection and chemoradiotherapy.

\section{CASE REPORT}

A 15-year-old girl was admitted to a local hospital because of a history of low-grade fever and fatigue, with right-side chest and back pain for more than half a year. Chest computed tomography (CT) showed signs of a huge tumor occupying the right chest wall, and the superior vena cava was likely to be full of tumor embolus.

Received February 27, 2021; received in revised form April 27, 2021; accepted April 27, 2021.

Correspondence: Xinjian Li, No. 59, Liuting St, Ningbo 315000, China; 13600626256 (e-mail: dxjs1961@sina.com); Chenwei Li, No. 59, Liuting St, Ningbo 315000, Cbina; 13819830649 (e-mail: nblcw2008@126.com).
The biopsy and pathology report showed osteosarcoma, which is known for its high fatality rate. The doctors of the local hospital advised the patient to be treated at a superior hospital. The patient came to the Oncology Department of our hospital to seek further diagnosis and treatment. After the patient was admitted to our department, she received physical examination, which showed jugular vein engorgement. The result showed that the sound of the left lung breath was clear, whereas that of the right lung breath was low. Dry and wet sounds were not heard in both lungs.

\section{Auxiliary Examination}

The echocardiography results showed abnormalities in the superior vena cava, right atrium, right ventricle, and pulmonary artery. The tricuspid valve was mildly closed. Enhanced CT scanning showed that the right side of the chest wall had a tumor that protruded out of the chest and affected the right atrial wall and right ventricular wall (Figure 1). The right side of the middle artery involved in papillary muscles was affected, and right-side pleural effusion was found locally. Upon bone scanning, the lesions were in the third to fifth right rib in accordance with the osteosarcoma. No obvious abnormality was found on the rest of bone scanning results. Cranial CT, electrocardiogram, routine ultrasound of the abdominal and lower limbs, and blood clotting and biochemical examinations showed no abnormalities.

After we fully communicated with the patient's family about the disease, we treated the tumor with neoadjuvant chemoradiotherapy consisting of $15 \mathrm{mg}$ Endostar (days 1 to 14) and $2 \mathrm{~g}$ ifosfamide (days 5 to 9). On the basis of the same chemotherapy, we added $120 \mathrm{mg}$ etoposide (days 5 to 9 ) with the former therapy as a second therapy after 3 weeks and a third therapy after 6 weeks. Surprisingly, after 3 courses of chemotherapy, the chest CT showed that the tumor in the fourth rib of the chest wall became smaller, and the tumor embolus in the superior vena cava was also smaller. Thus, we continued to treat the patient with another 3 courses of the same chemotherapy (each course interval was 21 days).

After 6 courses of chemotherapy, we provided radiotherapy (59.4 Gy/33 fractions) on the tumor at the right fourth rib. After neoadjuvant chemoradiotherapy, the chest CT showed that the tumor was located in the first anterior rib of the chest wall, with pleural invasion. Right pleural effusion formed a parcel, and the tumor embolus in the superior vena cava could hardly be seen. 


\section{Surgery}

After we evaluated the girl's physical condition, no obvious surgery contraindication could be found. Therefore, we decided to perform extracorporeal circulation and right chest wall tumor resection, right atrium tumor resection, and chest wall revascularization.

First, we prepared for extracorporeal circulation of the blood vessels. Intraoperatively, $30^{\circ}$ on the left side of the inclined decubitus, we made a longitudinal incision on the right groin separately to expose the right strands of the arteries and veins. Second, we made a posterior lateral incision to explore the chest wall tumor, whose diameter was approximately $15 \times 12 \times 10 \mathrm{~cm}$ (Figure 2). The tumor was qualitatively hard, with surrounding tissue adhesion and fully lesioned ribs. We performed truncation at approximately 2 $\mathrm{cm}$ on both ends of the neoplasm, with rib membrane excision (front end along the rib cartilage). Third, we started line heparinization on the femoral artery for blood vessels, double cavity drainage tube on the femoral vein, and internal jugular vein catheter puncture to establish extracorporeal circulation.

For the ultrasound-guided esophageal cut right atrium, we observed funicular, yellow, hard, basal parts connected to the atrial pectinate muscles probing up in the first vena cava, with unknown vein openings above. We also found funicular extension without the first vena cava. We cut the pulmonary trunk and found funicular material that extended to the left pulmonary artery bifurcation, which we fully explored after complete removal of the emboli.

Finally, for chest wall reconstruction, we performed extracorporeal circulation and chest wall reconstruction with a titanium plate $(15 \times 15 \mathrm{~cm})$ to repair the chest wall defect (Figure 3) by using steel wire with frame and rib cartilage suture fixation. Postoperative histopathology of the chest wall tumor showed that the source of rib bone sarcoma and right atrium floccule was the tumor emboli. The patient recovered after 16 days of treatment and continued to receive adjuvant chemotherapy. After postoperative follow-up of 6 months, the patient recovered perfectly, and no tumor recurrence or metastasis was found.

\section{DISCUSSION}

Osteosarcoma is one of most common sources of bone malignant tumors [Botchu 2006], whose tumor cells directly form osteoid tissues or bones. It tends to occur at the epiphysis, such as in the distal femoral and proximal tibia, of 11to 30-year-old individuals, but seldom occurs at the costa [Deitch 2003]. Comprehensive surgical treatment is the priority in treating osteosarcoma [Yaman 2017]. Rib osteosarcoma has a worse survival rate than long-bone osteosarcoma: its 5-year survival rate is only $15 \%$ [Suárez 2010].

In the case of rib osteosarcoma of our 15-year-old patient, the difficulty in surgical treatment lay in the following 3 points. First, the giant tumor was $15 \mathrm{~cm}$ in diameter and $900 \mathrm{~g}$ in weight. Second, the tumor emboli in the superior vena cava and the right atrium could detach at any time, which could lead to patient death from pulmonary embolism during the operation. Finally, reconstructing the line of the chest wall was necessary due to the large defects on the chest wall after tumor resection.

We first treated the huge tumor with 6 courses of chemotherapy and neoadjuvant radiotherapy to quickly narrow down the volume and provide good conditions for the operation. We decided auxiliary extracorporeal circulation was necessary during the operation to prevent the tumor emboli on

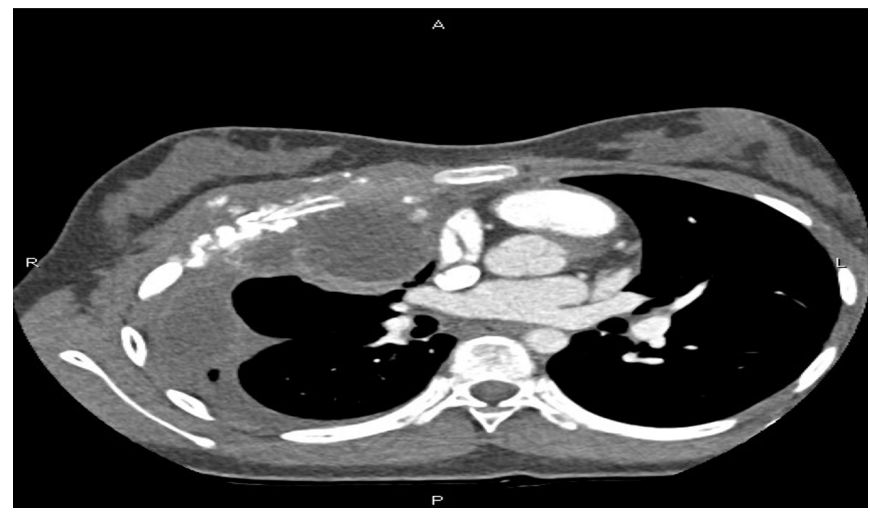

Figure 1. Preoperative chest wall CT, enhanced scanning.

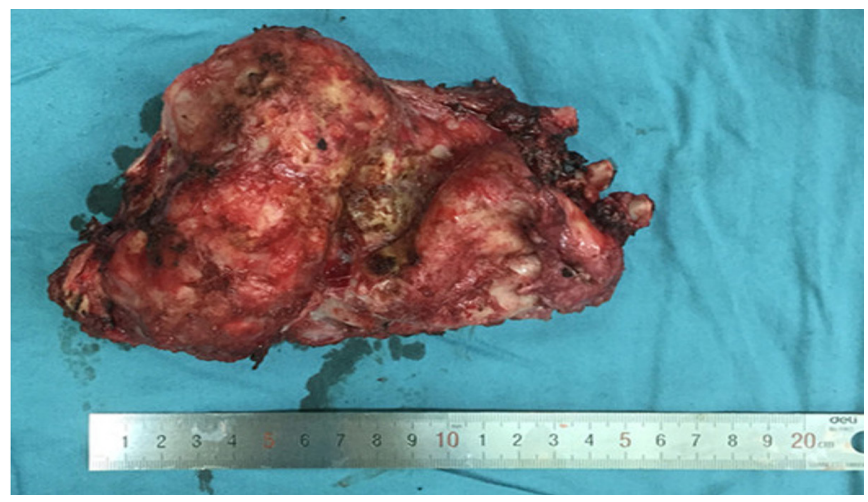

Figure 2. Surgical removal of giant tumor on chest wall.

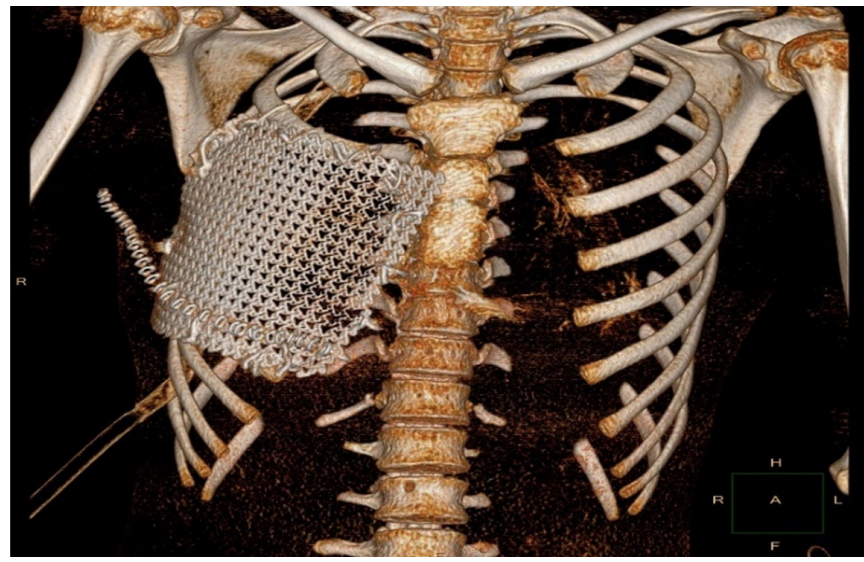

Figure 3. Postoperative chest wall reconstruction. 
the superior vena cava and the right atrium from detaching. If the tumor emboli did detach, we could cut the pulmonary artery and remove the emboli immediately. In addition, we added a titanium plate to fill the chest wall defect as a supplement. We treated the patient with more adjuvant chemotherapy as consolidation treatment to prevent tumor recurrence.

All in all, this case report demonstrated a new perspective for giant chest-wall osteosarcoma, which could not be surgically resected directly but required the help of neoadjuvant chemoradiation and auxiliary extracorporeal circulation. The procedures provided opportunities for complete resection and made it possible for the 15 -year-old patient to have quality long-term survival.

\section{ACKNOWLEDGMENTS}

We thank all members of our laboratories for useful discussions. This work was supported by grants from Ningbo Natural Science Foundation (no. 2018A610274) and Zhejiang Provincial Medicine and Health Technology Project (no. 2021PY067)

\section{REFERENCES}

Anoop TM, Geetha N, Babanrao SA, et al. Primary osteosarcoma of rib mimicking lung mass with secondary aneurysmal bone cyst formation. J
Thorac Oncol 2014;9:738-739.

Bacci G, Longhi A, Versari M, et al. Prognostic factors for osteosarcoma of the extremity treated with neoadjuvant chemotherapy: 15-year experience in 789 patients treated at a single institution. Cancer 2010;106:1154-1161.

Botchu R, Ravikumar KJ, Sudhakar G, et al. Osteosarcoma of rib in a seven-year-old child: A case report. Eur J Orthop Surg Traumatol 2006;16:156-157.

Deitch J, Crawford AH, Choudhury S. Osteogenic sarcoma of the rib: A case presentation and literature review. Spine 2003;28:74-77.

Haridas KL, Nair SG, Gopan G, et al. Uterine osteosarcoma with peritoneo-omental osteosarcomatosis: A case report and review of the literature. Ind J Gynecol Oncol 2020;18:26.

Ogundiran TO, Ademola SA, Oluwatosin OM, et al. Primary osteogenic sarcoma of the breast. World J Surg Oncol 2006;4:90.

Sabanathan S, Shah R, Mearns AJ. Surgical treatment of primary malignant chest wall tumours. Ann Thorac Surg 1997;60(suppl 1):S9.

Suárez AJ, Rodríguez GC, Montero MC, et al. Pulmonary Ewing sarcoma/primitive neuroectodermal tumor: A case report and a review of the literature. Archiv Bronconeumol 2010;46:44-46.

Yaman Bİ, Kurucu N, Oğuz B, et al. Primary osteosarcoma of the rib: A case report and review of the literature. J Pediatr Hematol Oncol 2018;40:48-50.

Zhang J, He X, Yu W, et al. Primary exophytic extraskeletal osteosarcoma of the liver: A case report and literature review. Risk Manag Healthc Policy 2021;14:1009-1014. 